\title{
Scottish Paediatric Society
}

At the Summer Meeting at Ninewells Hospital, Dundee, on 30 May 1975, the President, Dr. D. M. Douglas, was in the Chair.

The titles of the clinical demonstrations were as follows.

Congenital varicella from infection during second trimester. J. E. H. Brice (introduced). Ninewells Hospital, Dundee.

Stick injury of internal carotid artery. P. J. Smail (introduced). Ninewells Hospital, Dundee.

A boy with precocious puberty treated with cyproterone acetate. C. C. Forsyth. Ninewells Hospital, Dundee.

Septic pericarditis progressing to early constriction. R. A. F. Bell. Ninewells Hospital, Dundee.

Diagnosis of cystinosis. P. C. Etches (introduced). Ninewells Hospital, Dundee.

\section{Scientific communications}

Coliform meningitis in the newborn. J. $Z$. Heckmatt (introduced), and R. A. Shanks. Royal Hospital for Sick Children, Glasgow.

Between 1960 and 1974, 36 patients had coliform meningitis in the Special Care Baby Units of Queen Mother's Hospital, and Glasgow Royal Maternity Hospital, and in the Royal Hospital for Sick Children, Glasgow. 22 survived the initial illness and 14 died. Of the 22 survivors 8 died later, 7 of whom had hydrocephalus requiring drainage. The final number of survivors was therefore 14 . Esch. coli was the most common infecting organism. The 13 survivors who had been traced were well. Only 1 with a drain for hydrocephalus had survived. 3 survivors had some cranial enlargement but no other abnormality. The principal difficulties in initial diagnosis were the absence of clear clinical signs of meningitis and the not infrequent finding of blood-stained CSF from an apparently atraumatic lumbar puncture. The insidious onset and progression of the disease were evident in many cases; the need for vigilance with repeated cultures of urine, blood, and CSF was obvious. Comment on the success or failure of therapy was difficult because of the large number of antibiotics used in the study period. Chloramphenicol $50 \mathrm{mg} / \mathrm{kg}$ per day was the most widely used antibiotic in those who survived.
It was concluded that the diagnosis and management of coliform meningitis in the newborn are far from satisfactory and that a controlled therapeutic trial from more than one centre was needed.

Viral infections in asthma and wheezy bronchitis. I. Mitchell (introduced), H. Inglis (introduced), and H. Simpson. Royal Hospital for Sick Children, Edinburgh.

A 3-year prospective survey of the incidence of viral infections in children over the age of one year admitted to hospital with asthma and wheezy bronchitis (excluding acute bronchiolitis) was reported. Of 360 consecutive emergency admissions (192 first admissions, 168 readmissions), $267(74 \cdot 2 \%)$ had full clinical investigations and viral cultures. Paired sera were also examined in 75 of the latter. Of the 267 investigated, $141(52 \cdot 8 \%)$ were first admissions and $126(47 \cdot 2 \%)$ were readmissions. Viral infection was found in $\mathbf{4 6}$ $(17 \cdot 2 \%)$ of the 267 patients investigated, cultures being positive on 39 occasions. The most common viruses cultured were rhinovirus (16 admissions) and respiratory syncytial virus (12 admissions). The peak incidence of infection was in February and August. Children studied during second or subsequent admissions had a higher incidence of virus infection than those admitted for the first time $(P<0.01)$. Fewer girls than boys were admitted, but in children aged 5 years or more the isolation rate was higher in girls than boys $(P<0.05)$. The possible significance of these findings and their relation to published work was discussed.

Effective pulmonary capillary blood flow in infants with birth asphyxia. A. A. Hutchison (introduced) and G. Russell. University Department of Child Health, Foresterhill, Aberdeen.

The respiratory mechanics and effective pulmonary capillary blood flow ( $\dot{Q}_{P C}$ eff) in term infants with documented birth asphyxia were studied. Though no clinical or laboratory evidence of respiratory distress could be shown, all the infants had low values for $\dot{Q}_{P C}$ eff Birth asphyxia has been implicated in the aetiology of idiopathic respiratory distress syndrome and has been shown to be closely correlated with the severity and

TABLE

\begin{tabular}{|c|c|c|c|c|}
\hline & Birthweight (g) & $\begin{array}{l}\text { Gestational } \\
\text { age }(w)\end{array}$ & $\underset{\text { (ml/min) }}{\text { Q̀PC eff }}$ & $\begin{array}{c}\dot{\text { QPC eff }} \\
(\mathrm{ml} / \mathbf{k g} \text { per } \mathrm{min})\end{array}$ \\
\hline $\begin{array}{l}\text { Infants with birth asphyxia (20) } \\
\text { Normal infants (25) }\end{array}$ & $\begin{array}{l}3384 \\
3466\end{array}$ & $\begin{array}{l}40 \cdot 0 \\
40 \cdot 0\end{array}$ & $\begin{array}{l}405 \\
562\end{array}$ & $\begin{array}{l}121 \\
162\end{array}$ \\
\hline
\end{tabular}


prognosis of the condition. In addition, birth asphyxia is potentially avoidable and the importance of initial resuscitation in infants at risk of developing the respiratory distress syndrome has been stressed.

Recognition of hypertrophic obstructive cardiomyopathy in childhood. G. I. Fiddler (introduced) and M. J. Godman (introduced). Royal Hospital for Sick Children, Edinburgh.

Recent studies of hypertrophic obstructive cardiomyopathy have suggested that this is a familial condition transmitted as an autosomal dominant trait and may be associated with a poor long-term prognosis. The clinical diagnosis can be made in childhood in the presymptomatic phase, but may be difficult. 8 children with the condition between the ages of 1 and 13 years who were referred for evaluation of a late systolic murmur were discussed. Only one patient had symptoms. The clinical findings and electrocardiographic appearances in 5 suggested that the probable diagnosis was cardiomyopathy. In the remaining 3 the murmur was not thought to be diagnostic, and the electrocardiograms were normal. In 6 of the 8 patients cardiac catheterization and angiography confirmed the diagnosis. In all 8 patients, however, echocardiography showed the typical features of hypertrophic obstructive cardiomyopathy with asymmetrical hypertrophy of the interventricular septum and abnormal midsystolic reopening of the mitral valve. These observations confirmed that echocardiography is a valuable technique in the diagnosis of hypertrophic obstructive cardiomyopathy in childhood. Because it is noninvasive, the technique can be used to screen families of index cases. 